\title{
Quantitative Proteomic Analysis of the Metastasis-Inhibitory Mechanism of miR- 193a-3p in Non-Small Cell Lung Cancer
}

\author{
Wei Deng ${ }^{a}$ Mingxia Yan ${ }^{a}$ Tao Yu ${ }^{a}$ Haiyan Ge ${ }^{b}$ Hechun Lin ${ }^{a}$ Jing Lia \\ Ying Liu ${ }^{a}$ Qin Geng ${ }^{a}$ Miaoxin Zhu ${ }^{a}$ Lei Liu ${ }^{a}$ Xianghuo He Ming Yao $^{a}$ \\ aState Key Laboratory of Oncogenes and Related Genes, Shanghai Cancer Institute, Renji Hospital, \\ Shanghai Jiao Tong University School of Medicine, Shanghai, ${ }^{b}$ Propaganda Department, Huadong \\ Sanatorium, Wuxi, China
}

\author{
Key Words \\ MiR-193a-3p • NSCLC $\cdot$ iTRAQ $\cdot$ Proteome
}

\begin{abstract}
Background: microRNAs can repress the expression of target genes by destabilizing their mRNAs or by inhibiting their translation. Our previous findings suggested that miR-193a-3p inhibited the progression of NSCLC both in vitro and in vivo. However, the biological processes and molecular pathways through which this miRNA exerts its positive effects are unknown. Methods: To explore the molecular mechanisms by which miR-193a-3p inhibited NSCLC metastasis, we investigated the changes in the protein profile of SPC-A-1sci (highly metastatic) cells in response to the up-regulation of miR-193a-3p expression using a proteomics approach (iTRAQ combined with NanoLC-MS/MS). Changes in the profiles of the expressed proteins were verified using western blotting and were analyzed using the DAVID and STRING programs. Results: In the two replicated experiments, 4962/4946 proteins were identified, and the levels of expression of 4923/4902 proteins were quantified. In total, 112 of these proteins were differentially expressed. Among them, the up-regulated levels of expression of two of the 62 proteins with up-regulated expression (PPP2R2A and GSN) and the down-regulated levels of expression four of the 50 proteins with down-regulated expression (LMNB2, UHRF1, G3BP1, and HNRNPU) were verified using western blotting. The bioinformatics analysis revealed the interactions and signaling networks of these differentially expressed proteins. Conclusion: miR-193a-3p inhibited the metastasis of lung cancer cells by deregulating the expression of tumor-related proteins. These findings may improve the understanding of the molecular mechanisms underlying the metastatic-inhibitory effect of miR-193a-3p on lung cancer cells.
\end{abstract}

Copyright (C) 2015 S. Karger AG, Basel

W. Deng and M. Yan contributed equally to this work.

Ming Yao and

Xianghuo He
State Key Laboratory of Oncogenes and Related Genes,Shanghai Cancer Institute, Renji Hospital, Shanghai Jiao Tong University School of Medicine, No.25/Ln 2200 Xietu Road, Shanghai 200032 (China); E-Mail myao@shsci.org, and E-Mail xhhe@fudan.edu.cn 


\section{Introduction}

Lung cancer is the most prevalent cancer in humans and is the leading cause of cancerrelated deaths worldwide [1]. Although diagnostic and treatment methods have recently markedly improved, the 5 - and 10 -year survival rates remain at $<15 \%$ and $<7 \%$, respectively [2]. Lung cancer has the lowest survival outcomes due to the very high metastatic potential of these cancers. The majority of the mortalities due to lung cancers are attributable to tumor metastases [3]. Because the majority of lung cancer patients present with invasive, metastatic disease, it is vital to understand the molecular mechanisms underlying metastasis [4].

In our previous study, the highly metastatic cell line SPC-A-1sci was isolated from its parental SPC-A-1 cell line after several cycles of in vivo selection in NOD/SCID mice [5]. Proteomic approaches, which enable relatively comprehensive global analyses, have been widely used in studying complex biological functions [6, 7]. The isobaric tags for relative and absolute quantitation (iTRAQ) method combined with nano liquid chromatography-mass spectrometry (NanoLC-MS/MS), which was developed for protein quantitation, is a highthroughput quantitative technique [8, 9].

MicroRNAs (miRNA) are endogenous 23-nucleotide non-coding RNA molecules that regulate the expression protein-coding genes through degradation of the target mRNAs or inhibition of protein translation $[10,11]$. Emerging evidence shows that miRNAs are implicated in various biological processes, such as proliferation, apoptosis, cellular migration, and differentiation. It was demonstrated that dysregulation of miRNA expression caused abnormal gene expression in a variety of cancers $[12,13]$. In the field of miRNA research, the most important step is identifying the multiple target genes of miRNAs. Computational programs have been applied to predict the targets of miRNAs. However, these computational programs have inherent limitations, such as not considering the evidence for functional targeting within the 5'-untranslated region and protein-coding region [14-16] and not adjusting the predictions according to the co-expression of an miRNA and a target, which has been proposed to be an effective way of improving the accuracy of predictions [17], so that many of the predicted targets may be false and many genuine targets could be missed [18].

In the present study, with the aim of improving the understanding of the underlying molecular mechanisms underlying the metastasis-inhibitory effect of miR-193a-3p on lung cancer, a strategy that combined iTRAQ with NanoLC-MS/MS was utilized to analyze the alterations in the protein profile of the lung adenosquamous carcinoma cell line SPC-A-1sci following miR-193a-3p treatment.

\section{Materials and Methods}

\section{Cell lines and cell culture}

The highly metastatic human lung-cancer cell line SPC-A-1sci was established in our laboratory during a previous study through in vivo selection of clones of the cell line SPC-A-1, which has a low metastatic potential, grown in NOD/SCID mice [5]. The NSCLC cell line SPC-A-1sci was cultured in Dulbecco's Modified Eagle Medium (DMEM) supplemented with 10\% fetal bovine serum (FBS) (Biowest, South America Origin), $100 \mathrm{U} / \mathrm{mL}$ penicillin (Sigma-Aldrich, St. Louis, MO), and $100 \mu \mathrm{g} / \mathrm{mL}$ streptomycin (Sigma-Aldrich) in a humid chamber at $37^{\circ} \mathrm{C}$ in $5 \% \mathrm{CO}_{2}$.

Creation of stably transfected cell lines

The miRNA mimic and the negative control miRNA of hsa-miR-193a-3p and the lentiviral vector were purchased from the GenePharma Company. The sequence of the miRNA mimic was 5'-AACTGGCCTACAAAGTCCCAGT-3', and that of the miR-control was 5'-TTCTCCGAACGTGTCACGT-3'.

Stably transfected cell lines were generated by transfecting SPC-A-1sci cells with the miRNA mimic or the miR-control lentiviral vector. 


\section{Cellular Physiology Cell Physiol Biochem 2015;35:1677-1688 \begin{tabular}{l|l} 
and Biochemistry $\begin{array}{l}\text { DOI.1159/000373981 } \\
\text { Publisned onlne: Ivarch 18, } 2015\end{array}$ & $\begin{array}{l}\text { O 2015 S. Karger AG, Basel } \\
\text { www.karger.com/cpb }\end{array}$ \\
\cline { 2 - 3 }
\end{tabular} \\ Deng et al: Proteomic Analysis of MiR-193a-3p Targets in NSCLC Cells}

RNA Extraction and Quantitative Real-time PCR Assays

The total RNA was extracted from cultured cells using a mirVana ${ }^{\mathrm{TM}}$ miRNA isolation Kit (Ambion) according to the manufacturer's protocol. The concentration and purity of the RNA were determined using ultraviolet spectrophotometry using the Nanodrop 2000 system (Thermo, Japan). To evaluate the miR-193a$3 p$ expression levels, quantitative real-time PCR was performed using TaqMan miRNA assay kits (Applied Biosystems) according to the manufacturer's instructions. U6 mRNA was used as an endogenous reference, and its levels were used for normalization.

\section{Protein Preparation}

Harvested cells were washed three times using ice-cold PBS and were disrupted using a cell lysis buffer (Pierce, Rockford, IL, USA) containing protease and phosphatase inhibitors for $45 \mathrm{~min}$ on ice, followed by centrifugation at $12000 \times \mathrm{g}$ for $15 \mathrm{~min}$ at $4^{\circ} \mathrm{C}$. Next, the protein concentrations was determined using a bicinchoninic acid (BCA) protein assay kit according to the manufacturer's instructions (Pierce, Rockford, IL). Bovine serum albumin (BSA) was used as the standard.

\section{ITRAQ Labeling and High-pH RPLC Fractionation}

The iTRAQ Reagent 4-Plex kit (Applied Biosystems, Foster City, CA) was used according to the manufacturer's protocol to label peptides. Equal amounts of protein (100 $\mu \mathrm{g}$ per sample) obtained from the stably transfected cell lines (SPC-A-1sci NC and SPC-A-1sci miRNA mimic lines) were labeled using iTRAQ labeling reagents 116 and 117, respectively. Briefly, the proteins in each sample were reduced using tris-(2-carboxyethyl) phosphine (TCEP) and were alkylated using methyl methane-thiosulfonate (MMTS). Afterward, the samples were digested overnight at $37^{\circ} \mathrm{C}$ using trypsin (mass spectrometry grade, Promega, Madison, WI) at a trypsin:protein ratio of 1:20 (W/W). Then, the tryptic peptides were labeled using iTRAQ reagents. The iTRAQ-labeled samples were combined in a fresh tube, desalted using Oasis HLB cartridges (Waters, Milford, MA, USA) and dried using a vacuum centrifuge (Concentrator Plus, Eppendorf, Germany). The iTRAQ-labeled peptides were fractionated using an Agilent 1290 system using a Durashell C18 column $(4.6 \times 250 \mathrm{~mm}, 5 \mu \mathrm{m}, 100 \AA$, Agela technologies, China) for high-pH RP chromatography. A total of $20 \mathrm{RP}$ fractions were collected and were then desalted using C18 cartridges (UltraMicroSpin, The Nest Group, Southborough, MA), after which they were dried and were reconstituted using $20 \mu \mathrm{L}$ of $0.1 \%$ FA for NanoLCMS/MS analysis.

\section{NanoLC-MS/MS Analysis}

The RP fractions were analyzed in duplicate using NanoLC-MS/MS using a NanoLC system (NanoLC2D Ultra, Eksigent, Dublin, CA) connected to a Triple TOF 5600 mass spectrometer (AB SCIEX, USA). Briefly, the peptides were enriched on an RP Trap column (ProteoPepIIC18 column, $5 \mu \mathrm{m}, 300 \AA, 0.15 \times 25 \mathrm{~mm}$, IntegraFrit, New Objective, Woburn, MA) and were then eluted onto an analytical column (ProteoPepC18 column, $5 \mu \mathrm{m}, 300 \AA$, $0.075 \times 150 \mathrm{~mm}$, IntegraFrit, Woburn, MA) and were separated using a 120-min gradient of 5 to $35 \%$ Buffer B (98\% ACN, 2\% H2O, 0.1\% FA) at a flow rate of $300 \mathrm{~nL} / \mathrm{min}$. The MS analysis was performed using a nano ion spray voltage that was typically maintained at $2.3 \mathrm{kV}$ and a scan range of 350 to 1500 (m/z) in the positive-ion mode. Full-scan MS spectra were acquired from 40 precursors that had been selected for MS/MS from the m/z 100-1500 range using a dynamic exclusion setting of $30 \mathrm{~s}$. The IDA CE parameter script, which selected up to 40 precursors with charge states of $2+$ to $4+$, automatically controlled the collision energy (CE). The mass spectrometer was calibrated using the tryptic peptides of a beta-galactosidase (BG).

\section{Protein Identification and Quantitation}

Analysis of the proteins was performed using ProteinPilot4.1 software (AB SCIEX, USA). The search parameters were as follows: (1) sample type, iTRAQ 4-plex (Peptide Labeled); (2) cysteine alkylation, MMTS; (3) digestion, trypsin; (4) instrument, Triple T0F5600; (5) special factors, none; (6) species, Homo sapiens; (7) ID Focus, biological modifications; (8) database, UniProtKB/Swiss-Prot FASTA (which was released in November, 2013 and consists of 176592 human sequences); and (9) search effort, Thorough ID. In the iTRAQ quantitation, the Pro Group algorithm had been automatically selected to calculate the reporter-peak areas. To estimate the FDR for peptide identification, a decoy database search strategy was adopted. For this study, a strict unused confidence score of $>1.3$ were used as the qualification criteria, which corresponded to 
a peptide confidence level of $95 \%$. If the iTRAQ ratios were $>1.5$ or $<0.67$ in the samples obtained from the highly metastatic SPC-A-1sci cells containing miR-193a-3p relative to those of the miR-control, the proteins were considered to be differentially expressed.

\section{Western Blotting (WB)}

Protein samples of the stably transfected cell lines SPC-A-1sci miR-control and SPC-A-1sci miRNA mimic were harvested. Twenty micrograms of protein/well was loaded on $8 \%$ gels for separation using sodium dodecyl sulfate-polyacrylamide gels electrophoresis (SDS-PAGE) and subsequent electroblotting onto NC membranes (Millipore, Billerica, MA, USA). The membranes were then blocked for one hour using 5 $\%$ nonfat dry milk in PBS-T ( $0.05 \%$ Tween-20 in PBS). After blocking, the membranes were rinsed and were incubated with rabbit anti-PPP2R2A (1:100), rabbit anti-G3BP1 (1:500) and rabbit anti-UHRF1 (1:250) antibodies purchased from Proteintech ${ }^{\mathrm{TM}}$; rabbit anti-GSN (1:500) antibodies purchased from Cell Signaling Technology and rabbit anti-HUNRNPU (1:50) antibodies purchased from Abcam; mouse anti-LMNB2 (1:20) antibodies purchased from Santa Cruz Biotechnology. HRP-conjugated anti-goat IgG (1:20000) purchased from Sigma-Aldrich was used as a secondary antibody for an incubation period of $1 \mathrm{~h}$. The labeled bands were visualized using the SuperSignal West Femto Maximum Sensitivity Substrate (Thermo, Japan), and the $\beta$-actin levels were used as the loading controls.

\section{Data Analysis}

STRING (http://string.embl.de) is a database of predicted signaling networks and protein interactions. Ontological analysis of the dysregulated genes was performed using the search tool DAVID (http://david. abcc.ncifcrf.gov/).

\section{Statistical Analysis}

The levels of miR-193a-3p expression, determined using real-time PCR, and the differences in the protein profiles of the groups of cells were compared using the t-test, using SPSS 19.0 software. A P-value of less than 0.05 was considered significant.

\section{Results}

Comparative Proteomics of SPC-A-1sci miRNA mimic Cells versus SPC-A-1sci miR-control Cells

We created stably transfected cell lines by transfecting SPC-A-1sci cells with the miRNA mimic/miR-control lentiviral vectors. The extracted RNA was used to detect the levels of miR-193a-3p expression (Fig. 1A). The proteins were extracted from the two cell lines. The samples were digested using trypsin and labeled using the 116 and 117 iTRAQ tags, respectively. The labeled digests were utilized for MS analysis (Fig. 1B). Using ProteinPilot 4.1 , we identified a total of $6127 / 6118$ proteins in these two cell lines (global FDR of $<1 \%$ ). Using the filters of an unused protein score of $>1.3$ and a number of peptides of $\geq 2,4962 / 4946$ proteins were identified and the amounts of $4923 / 4902$ proteins were quantified. The labelling rate was $99.2 \%(4923 / 4962)$ and $99.1 \%(4902 / 4946)$ in the two runs (Table 1). In total, we identified 62 up-regulated (with 117:116 iTRAQ ratios of $>1.5$ in the two runs) and 50 down-regulated proteins (with 117:116 iTRAQ ratios of $<0.67$ in the two runs) in SPC-A-1sci miRNA mimic cells compared with the SPC-A-1sci miR-control cells (Fig. 1C and Tables 2 and 3 ).

Table 1. Protein numbers of indentified and quantitative by iTRAQ

\begin{tabular}{ccccc}
\hline $\begin{array}{c}\text { Experiment } \\
\text { N0. }\end{array}$ & $\begin{array}{c}\text { Lobal } \\
\text { FDR }<1 \%\end{array}$ & $\begin{array}{c}\text { Unused protein score }(>1.3) \\
\text { and peptides }(\geq 2)\end{array}$ & $\begin{array}{c}\text { Labled } \\
\text { protein }\end{array}$ & lable ratio \\
\hline 1 & 6127 & 4962 & 4923 & $99.20 \%$ \\
2 & 6118 & 4946 & 4902 & $99.10 \%$ \\
\hline
\end{tabular}


Fig. 1. Experimental workflow for the quantitative proteomic analysis and the results. (A) SPC-A-1sci cells were transfected with a miR-control lentiviral vector or a miR-193a-3pmimic lentiviral vector. The miRNA was extracted from cultured cells using the mirVana miRNA Isolation Kit (Ambion, Carlsbad, CA, USA) and was quantified using probes in TaqMan miRNA assays (Applied Biosystems, Carlsbad, CA, USA) performed according to the manufacturer's instructions. (B) The proteins of the cell lines that were stably transfected with the miR-control and the miR-mimic were digested with trypsin and were labeled using 116/117 ITRAQ tags, respectively. The labeled digests were separated using offline SCX LC and were analyzed using Nano LCMS/MS. The differentially expressed proteins were evaluated using western blotting and were analyzed through database searches. (C) In total, 112 proteins were identified, including 62 up-regulated proteins and 50 down-regulated proteins, in two physical replicates.

\section{Cluster Analysis of the miR-193a-3p-regulated Proteins}

The heatmap contains the data obtained for the 112 dysregulated proteins. The two horizontal clusters represent the technical replicates. The clustering of the globally dysregulated proteins in the miR-193a-3p-overexpressing cells may provide direct evidence that the genes encoding these differentially expressed proteins are regulated by miR-193a3p (Fig. 2).

\section{Evaluation of iTRAQ Results using Western Blotting}

Based on the results of the MS analysis, the levels of expression of six of the dysregulated proteins was validated using western blotting analysis of proteins of the SPC-A-1sci mir193a-3p mimic cells and the SPC-A-1sci miRNA negative control cells. The expression levels of four proteins (HNRNPU, G3BP1, UHRF1, and LMNB2) were down-regulated (Fig. 3A) and the expression levels of two proteins (PPP2R2A and GSN) were up-regulated (Fig. 3B), which was consistent with the results of the MS analysis. Therefore, the expression levels of 112 proteins were considered to be regulated by miR-193a-3p.

\section{Functional Enrichment of the miR-193a-3p-regulated Proteins}

Some of the proteins among the globally dysregulated group have been reported to be involved in lung cancer; for example, UHRF1, G3BP1 and KRT7 were associated with lung cancer metastasis and PSAT1, PRKDC and PPP2R2A were associated with lung-cancer cell proliferation. To further elucidate the biological processes affected by miR-193a-3p expression, these differentially expressed proteins were applied to the DAVID database to achieve an enrichment analysis regarding involvement in a biological process, a cellular component or a molecular function (Fig. 4).

\section{Analysis of the signaling network}

We uploaded the globally dysregulated proteins after stably transfecting the miR-193a$3 p$ and their names into the STRING database. Ninety-four proteins were identified by the 
Table 2. List of up-regulated proteins

database, whereas 24 proteins did not show any link at the chosen confidence level (STRING score $=0.4$ ). A merged network is shown in Fig. 5. The most significant functions of the proteins in the network were cytoskeletal organization, RNA transport, RNA processing and protein localization. KHSRP, RANBP2, XPO1, LRPPRC and TPR are important proteins in RNA transport. DDX23, DHX9, KHSRP, SSB, HNRNPR and HNRNPU are important proteins in the RNA metabolic process. ACTN4, GSN, KRT19, KRT8 and PAFAH1B1 are associated with cytoskeletal organization. The program predicted associations for a particular group of proteins.

\section{Discussion}

In a previous study, we demonstrated that miR-193a-3p was involved in metastasis of NSCLC cells. Overexpression of miR-193a-3p suppressed the migration and invasion of NSCLC cells in vitro and NSCLC metastasis in vivo through down-regulating the expression of ERBB4 and S6K2 [19]. However, the complex cancer inhibitory mechanisms

\begin{tabular}{|c|c|c|c|c|c|c|}
\hline Accession NO. & $\begin{array}{l}\text { Protein } \\
\text { name }\end{array}$ & $\begin{array}{l}\text { Unused score } \\
\text { (mean } \pm S D \text { ) }\end{array}$ & $\begin{array}{l}\% \operatorname{Cov}[95) \\
\text { (mean } \pm S D \text { ) }\end{array}$ & $\begin{array}{c}\text { Peptides(95\%) } \\
\text { (mean } \pm \text { SD) }\end{array}$ & $\begin{array}{l}\text { iTRAQ Rate } \\
\text { (mean } \pm S D \text { ) }\end{array}$ & $\begin{array}{c}\text { Pval } \\
\text { (mean } \pm S D)\end{array}$ \\
\hline A0MZ66 & KIAA1598 & $26.11 \pm 2.47$ & $39.3 \pm 1.84$ & $18 \pm 3$ & $3.12 \pm 0.22$ & $0.01 \pm 0$ \\
\hline 000159-2 & MY01C & $40.08 \pm 3.67$ & $39.25 \pm 2.19$ & $25 \pm 4$ & $2.94 \pm 0.15$ & $0 \pm 0$ \\
\hline 000299 & CLIC1 & $37.97 \pm 4.67$ & $83 \pm 0$ & $46 \pm 4$ & $2.79 \pm 1.05$ & $0 \pm 0$ \\
\hline 014929 & HAT1 & $15.57 \pm 0.96$ & $35.8 \pm 5.8$ & $15 \pm 1$ & $1.9 \pm 0.11$ & $0.05 \pm 0$ \\
\hline 015031 & PLXNB2 & $13.4 \pm 1.18$ & $20.4 \pm 4.24$ & $11 \pm 0$ & $2.21 \pm 0.6$ & $0.01 \pm 0$ \\
\hline 043707 & ACTN4 & $112.36 \pm 0.24$ & $80.75 \pm 2.76$ & $142 \pm 6$ & $2.88 \pm 0.04$ & $0 \pm 0$ \\
\hline 043795-2 & MY01B & $17.49 \pm 1.9$ & $24.8 \pm 2.55$ & $11 \pm 2$ & $1.88 \pm 0.29$ & $0.02 \pm 0.01$ \\
\hline 075489 & NDUFS3 & $10.81 \pm 1.07$ & $38.45 \pm 2.9$ & $7 \pm 1$ & $2.93 \pm 0.49$ & $0.02 \pm 0.01$ \\
\hline 075521 & ECI2 & $15.04 \pm 1.36$ & $41.8 \pm 1.98$ & $11 \pm 3$ & $2 \pm 0.65$ & $0.04 \pm 0$ \\
\hline P02786 & TFRC & $38.81 \pm 5.29$ & $47.3 \pm 5.09$ & $25 \pm 3$ & $2.86 \pm 0.52$ & $0 \pm 0$ \\
\hline P04264 & KRT1 & $15.67 \pm 1.92$ & $43 \pm 1.27$ & $16 \pm 1$ & $2.97 \pm 1.08$ & $0.01 \pm 0.01$ \\
\hline P05023-3 & ATP1A1 & $42.28 \pm 4.08$ & $39.65 \pm 1.48$ & $39 \pm 1$ & $4.17 \pm 1.74$ & $0 \pm 0$ \\
\hline P05455 & SSB & $35.82 \pm 1.5$ & $51.5 \pm 8.34$ & $24 \pm 3$ & $2.43 \pm 0.35$ & $0.03 \pm 0.03$ \\
\hline P06396 & GSN & $32.01 \pm 3.8$ & $49.05 \pm 5.02$ & $27 \pm 2$ & $2.08 \pm 0.39$ & $0 \pm 0$ \\
\hline P06576 & ATP5B & $41.44 \pm 0.9$ & $59.85 \pm 4.45$ & $45 \pm 10$ & $2.85 \pm 0.61$ & $0 \pm 0$ \\
\hline P06756-3 & ITGAV & $30.43 \pm 6.36$ & $34.55 \pm 3.18$ & $20 \pm 4$ & $2.08 \pm 0.15$ & $0.01 \pm 0$ \\
\hline P07339 & CTSD & $36.13 \pm 2.38$ & $61.3 \pm 0.14$ & $37 \pm 4$ & $2.68 \pm 0.81$ & $0 \pm 0$ \\
\hline P08758 & ANXA5 & $67.26 \pm 5.41$ & $88.75 \pm 1.34$ & $73 \pm 4$ & $2.54 \pm 0.44$ & $0.01 \pm 0.01$ \\
\hline P09960 & LTA4H & $36.83 \pm 3.68$ & $48 \pm 0$ & $39 \pm 2$ & $1.59 \pm 0.04$ & $0.03 \pm 0.02$ \\
\hline P10515 & DLAT & $18.97 \pm 3$ & $31 \pm 1.84$ & $14 \pm 1$ & $6.33 \pm 4.68$ & $0 \pm 0$ \\
\hline P11177 & PDHB & $22.13 \pm 4.54$ & $43.3 \pm 4.53$ & $22 \pm 1$ & $2.89 \pm 0.11$ & $0.01 \pm 0$ \\
\hline P14625 & HSP90B1 & $93.88 \pm 4.55$ & $68.7 \pm 1.13$ & $97 \pm 1$ & $1.61 \pm 0.05$ & $0 \pm 0$ \\
\hline P22314 & UBA1 & $94.08 \pm 13.89$ & $69.75 \pm 5.3$ & $119 \pm 4$ & $2.98 \pm 0.14$ & $0.01 \pm 0.01$ \\
\hline P25705 & АТP5A1 & $48.12 \pm 5.03$ & $54.35 \pm 3.46$ & $40 \pm 2$ & $2.86 \pm 0.19$ & $0 \pm 0$ \\
\hline P26038 & MSN & $64.67 \pm 4.27$ & $71.05 \pm 7.85$ & $90 \pm 7$ & $1.71 \pm 0.03$ & $0.02 \pm 0.01$ \\
\hline P30041 & PRDX6 & $33.43 \pm 0.87$ & $87.75 \pm 0.35$ & $40 \pm 1$ & $5.07 \pm 1.05$ & $0 \pm 0$ \\
\hline P30101 & PDIA3 & $53.19 \pm 4.97$ & $71.4 \pm 2.69$ & $63 \pm 1$ & $4.54 \pm 1.14$ & $0 \pm 0$ \\
\hline P31327 & CPS1 & $90.32 \pm 5.06$ & $49.3 \pm 3.39$ & $78 \pm 6$ & $3.08 \pm 0.32$ & $0.01 \pm 0$ \\
\hline P33992 & MCM5 & $47.41 \pm 0.08$ & $53.1 \pm 0.57$ & $36 \pm 2$ & $2.11 \pm 0.03$ & $0.01 \pm 0$ \\
\hline P35221 & CTNNA1 & $50.52 \pm 2.64$ & $48.95 \pm 2.47$ & $35 \pm 2$ & $2.63 \pm 0.36$ & $0 \pm 0$ \\
\hline P38117 & ETFB & $13.57 \pm 0.51$ & $45.9 \pm 1.13$ & $8 \pm 1$ & $2.33 \pm 0.03$ & $0.03 \pm 0.01$ \\
\hline P40925-3 & MDH1 & $27.13 \pm 0.02$ & $61.1 \pm 2.4$ & $21 \pm 0$ & $1.72 \pm 0.13$ & $0.01 \pm 0$ \\
\hline P40939 & HADHA & $44.79 \pm 1.7$ & $50.6 \pm 2.26$ & $31 \pm 3$ & $2.18 \pm 0.56$ & $0 \pm 0$ \\
\hline P42704 & LRPPRC & $81.88 \pm 0.11$ & $53.15 \pm 1.2$ & $77 \pm 1$ & $5.02 \pm 0.81$ & $0 \pm 0$ \\
\hline P43034 & PAFAH1B1 & $17.51 \pm 1.35$ & $42.2 \pm 0.71$ & $15 \pm 2$ & $2.79 \pm 0.36$ & $0.03 \pm 0.02$ \\
\hline P46939 & UTRN & $38.57 \pm 3.13$ & $22.85 \pm 4.74$ & $26 \pm 4$ & $3.32 \pm 1.14$ & $0 \pm 0$ \\
\hline P46940 & IQGAP1 & $149.37 \pm 4.07$ & $62.3 \pm 0.28$ & $154 \pm 0$ & $2.56 \pm 0.23$ & $0 \pm 0$ \\
\hline P49736 & MCM2 & $47.28 \pm 0.59$ & $45.25 \pm 1.06$ & $39 \pm 5$ & $2.29 \pm 0.09$ & $0.01 \pm 0$ \\
\hline P54709 & ATP1B3 & $14.07 \pm 0.64$ & $37.25 \pm 6.58$ & $9 \pm 1$ & $4.69 \pm 0.46$ & $0.01 \pm 0.01$ \\
\hline P60842 & EIF4A1 & $57.16 \pm 2.45$ & $82.75 \pm 1.34$ & $76 \pm 1$ & $3.83 \pm 0.87$ & $0.03 \pm 0$ \\
\hline P63151 & PPP2R2A & $30.65 \pm 2.21$ & $61.85 \pm 4.6$ & $24 \pm 2$ & $2.07 \pm 0.49$ & $0.03 \pm 0$ \\
\hline P80303 & NUCB2 & $14.87 \pm 1.23$ & $42 \pm 5.23$ & $11 \pm 1$ & $2.81 \pm 0.46$ & $0 \pm 0$ \\
\hline Q02218 & OGDH & $34.94 \pm 1.26$ & $32.4 \pm 0.57$ & $24 \pm 1$ & $2.52 \pm 0.36$ & $0 \pm 0$ \\
\hline Q02952-3 & AKAP12 & $30.86 \pm 3.98$ & $34.55 \pm 7$ & $18 \pm 4$ & $6.68 \pm 2.1$ & $0 \pm 0$ \\
\hline Q14697 & GANAB & $52.88 \pm 0.88$ & $49.45 \pm 1.63$ & $39 \pm 1$ & $4.86 \pm 2.64$ & $0 \pm 0$ \\
\hline Q15147 & PLCB4 & $15.25 \pm 1.73$ & $22.05 \pm 3.75$ & $9 \pm 1$ & $1.95 \pm 0.06$ & $0.03 \pm 0.01$ \\
\hline Q6PID6 & TTC33 & $8.01 \pm 0.01$ & $29.4 \pm 1.13$ & $6 \pm 1$ & $2.94 \pm 0.79$ & $0.03 \pm 0.01$ \\
\hline Q86VS8 & HOOK3 & $18.26 \pm 0.07$ & $33.05 \pm 5.73$ & $12 \pm 0$ & $2.45 \pm 0.43$ & $0.01 \pm 0.01$ \\
\hline Q96199 & SUCLG2 & $19.76 \pm 1.82$ & $45.25 \pm 0.49$ & $15 \pm 1$ & $2.41 \pm 0.34$ & $0.02 \pm 0.02$ \\
\hline Q99436 & PSMB7 & $23.82 \pm 1.9$ & $56.7 \pm 13.29$ & $17 \pm 2$ & $2.51 \pm 0.93$ & $0.03 \pm 0.01$ \\
\hline
\end{tabular}

continued 
underlying the effects of this small non-coding RNA and the molecular targets through which miR-193a-3p acts have not yet been fully determined.

In this study, the lung adenocarcinoma cancer cell line SPC-A1 sci, which was established by our laboratory and which has a highly metastatic potential, was used as a model, and the isobaric tags for relative and absolute quantitation (iTRAQ) approach combined with nano liquid chromatography-tandem mass spectrometry (NanoLC-MS/MS) analysis were used to identify the proteins species with altered levels of expression in the SPC-A-1sci cells that overexpressed miR-193a-3p. The proteomic analysis revealed a total of 112 dysregulated protein species, the functions of which were involved in diverse biological processes, such as protein transport, RNA processing, cytoskeletal organization and translation. Among these proteins, 62 proteins exhibited up-regulated expression and 50 proteins exhibited down-regulated expression. The oncoproteins with miR-193a-3p-dysregulated expression are thought to be associated with the inhibition of NSCLC progression.

GSN and PPP2R2A were up-regulated by miR-193a-3p. Gelsolin (GSN) is a cytoskele-

\begin{tabular}{llllccc}
\hline Accession NO. & $\begin{array}{l}\text { Protein } \\
\text { name }\end{array}$ & $\begin{array}{l}\text { Unused score } \\
(\text { mean } \pm \text { SD })\end{array}$ & $\begin{array}{l}\text { \% } \operatorname{Cov}(95) \\
\text { (mean } \pm \text { SD })\end{array}$ & $\begin{array}{c}\text { Peptides(95\%) } \\
\text { (mean } \pm \text { SD })\end{array}$ & $\begin{array}{c}\text { iTRAQ Rate } \\
(\text { mean } \pm \text { SD })\end{array}$ & $\begin{array}{c}\text { Pval } \\
\text { (mean } \pm S D)\end{array}$ \\
\hline Q9BVG4 & PBDC1 & $12.15 \pm 0.18$ & $51.3 \pm 7.64$ & $9 \pm 1$ & $1.96 \pm 0.24$ & $0.04 \pm 0$ \\
Q9BXJ9 & NAA15 & $31.07 \pm 1.41$ & $42.7 \pm 0$ & $21 \pm 5$ & $2.09 \pm 0.16$ & $0.02 \pm 0.02$ \\
Q9H074 & PAIP1 & $12.38 \pm 3.02$ & $34.45 \pm 5.02$ & $10 \pm 2$ & $2.54 \pm 0.2$ & $0.03 \pm 0.01$ \\
Q9NQR4 & NIT2 & $23.42 \pm 0.83$ & $58.7 \pm 0.57$ & $14 \pm 0$ & $2.59 \pm 0.24$ & $0 \pm 0$ \\
Q9NT62 & ATG3 & $15.1 \pm 0.74$ & $45.05 \pm 7.85$ & $12 \pm 0$ & $2.06 \pm 0.24$ & $0.04 \pm 0$ \\
Q9UBT2 & UBA2 & $28.41 \pm 4.61$ & $47.5 \pm 0.85$ & $22 \pm 1$ & $1.77 \pm 0.05$ & $0.01 \pm 0.01$ \\
Q9UKY7 & CDV3 & $14.97 \pm 1.24$ & $73.1 \pm 0.28$ & $11 \pm 2$ & $5.15 \pm 2.51$ & $0.01 \pm 0.01$ \\
Q9ULZ3 & PYCARD & $5.37 \pm 0.9$ & $23.3 \pm 2.55$ & $4 \pm 0$ & $10.5 \pm 4.32$ & $0.03 \pm 0.01$ \\
B4DWW4 & MCM3 & $56.44 \pm 3.22$ & $56.1 \pm 1.13$ & $46 \pm 6$ & $3.53 \pm 1.02$ & $0 \pm 0$ \\
C9JIZ6 & PSAP & $30.06 \pm 4.87$ & $46.9 \pm 2.55$ & $27 \pm 1$ & $7.04 \pm 3.55$ & $0.03 \pm 0.01$ \\
E9PRY8 & EEF1D & $36.65 \pm 0.62$ & $48.15 \pm 2.33$ & $40 \pm 2$ & $3.58 \pm 1.58$ & $0.02 \pm 0.01$ \\
K7ELL7 & PRKCSH & $32.53 \pm 0.54$ & $57.6 \pm 1.84$ & $32 \pm 4$ & $3 \pm 1.44$ & $0.01 \pm 0.01$ \\
\hline
\end{tabular}

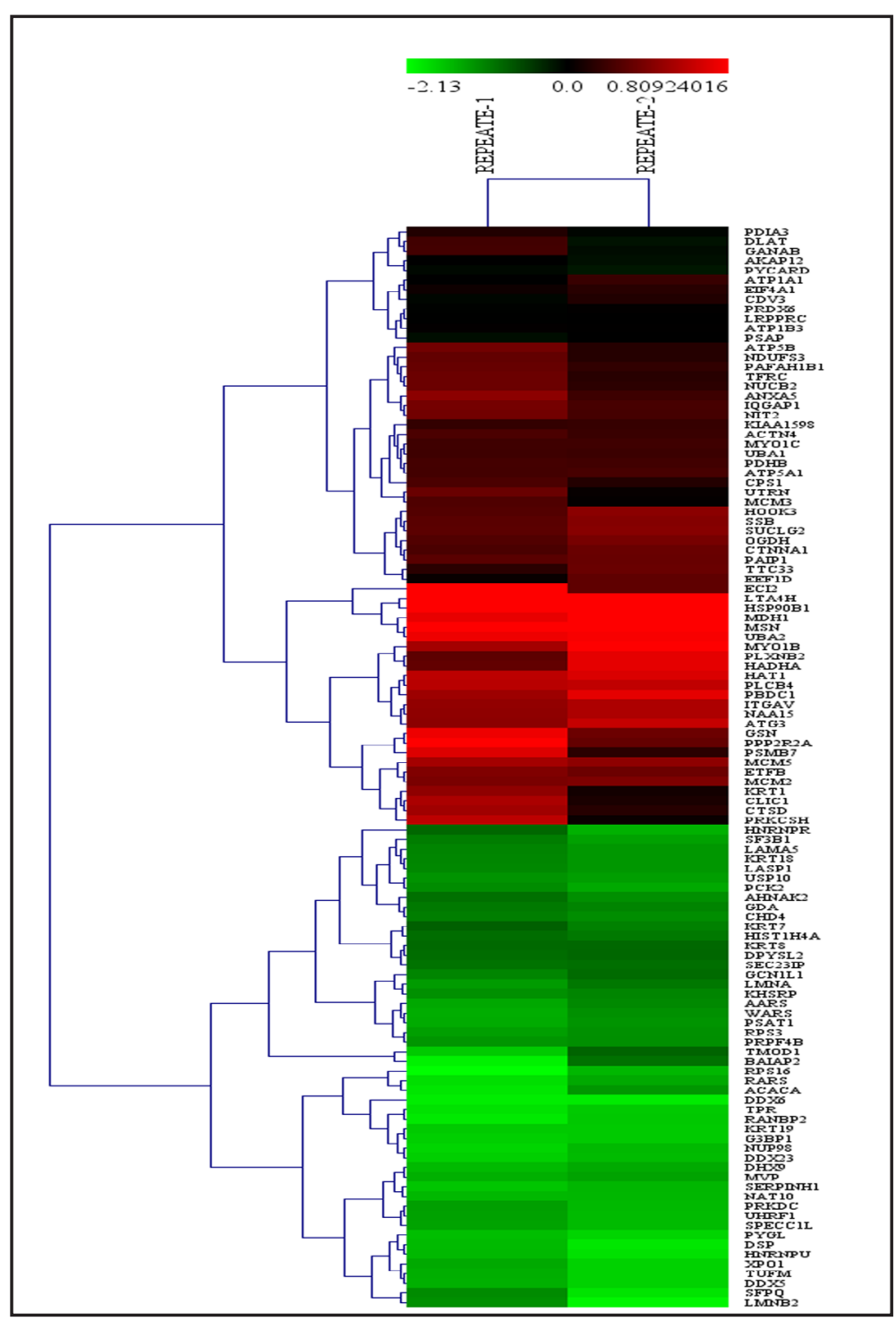

Fig. 2. The expression levels of the 112 dysregulated proteins are shown in a Heatmap. The red-colored clusters represent up-regulated proteins and the green-colored clusters represent down-regulated proteins. 
Table 3. List of downregulated proteins

ton-associated protein that is involved in dynamic changes in the actin cytoskeleton during cell motility [20]. It has been reported that GSN is aberrantly expressed in many types of tumor. Under various pathological conditions and in different types of cancer cells, GSN performs different functions. For example, high GSN expression in oral cancers is related to a poor clinical outcome for patients with metastasis [21]. In contrast, GSN expression is frequently down-regulated in ovarian, stomach and colon cancers [2224]. In our study, the up-regulation of GSN expression may play a role in inhibiting the metastasis of SPC-A1 sci cells transfected with miR-193a-3p. PPP2R2A is a candidate tumor-suppressor gene that is implicated in the negative control of cell growth and division because it is a member of the protein phosphatase 2 (PP2A) regulatory subunit B family [25]. Many studies have reported that PPP2R2A down-regulation was observed in acute

\begin{tabular}{|c|c|c|c|c|c|c|}
\hline Accession NO. & $\begin{array}{l}\text { Protein } \\
\text { name }\end{array}$ & $\begin{array}{l}\text { Unused score } \\
\text { (mean } \pm S D \text { ) }\end{array}$ & $\begin{array}{l}\% \operatorname{Cov}(95) \\
(\text { mean } \pm S D)\end{array}$ & $\begin{array}{c}\text { Peptides(95\%) } \\
\text { (mean } \pm \text { SD) }\end{array}$ & $\begin{array}{l}\text { iTRAQ Rate } \\
(\text { mean } \pm S D)\end{array}$ & $\begin{array}{c}\text { Pval } \\
(\text { mean } \pm S D)\end{array}$ \\
\hline 014980 & ХР01 & $58.64 \pm 3.42$ & $46.4 \pm 0$ & $57 \pm 1$ & $0.54 \pm 0.06$ & $0 \pm 0$ \\
\hline 015230 & LAMA5 & $31.42 \pm 2.06$ & $15.25 \pm 0.64$ & $19 \pm 1$ & $0.39 \pm 0.04$ & $0 \pm 0$ \\
\hline 043390 & HNRNPR & $12.93 \pm 0.48$ & $33.2 \pm 3.82$ & $16 \pm 1$ & $0.36 \pm 0.21$ & $0.01 \pm 0.02$ \\
\hline 075533 & SF3B1 & $47.4 \pm 0.75$ & $41.15 \pm 2.33$ & $32 \pm 1$ & $0.4 \pm 0.09$ & $0 \pm 0$ \\
\hline P02545 & LMNA & $49.59 \pm 2.02$ & $53.95 \pm 4.88$ & $40 \pm 4$ & $0.37 \pm 0.12$ & $0 \pm 0$ \\
\hline P05783 & KRT18 & $61.98 \pm 0.13$ & $81.4 \pm 3.96$ & $75 \pm 8$ & $0.4 \pm 0.03$ & $0 \pm 0$ \\
\hline P05787 & KRT8 & $77.17 \pm 3.97$ & $78.2 \pm 0.14$ & $93 \pm 12$ & $0.21 \pm 0.03$ & $0 \pm 0$ \\
\hline P06737 & PYGL & $35.53 \pm 8.95$ & $47.35 \pm 4.17$ & $31 \pm 2$ & $0.57 \pm 0.03$ & $0.02 \pm 0.02$ \\
\hline P08727 & KRT19 & $31.49 \pm 1.28$ & $72.3 \pm 2.83$ & $25 \pm 0$ & $0.58 \pm 0.01$ & $0.01 \pm 0.01$ \\
\hline P08729 & KRT7 & $41.59 \pm 1.55$ & $65.15 \pm 6.15$ & $51 \pm 1$ & $0.25 \pm 0.11$ & $0 \pm 0$ \\
\hline P12270 & $\mathrm{TPR}$ & $67.47 \pm 1.46$ & $35.15 \pm 1.06$ & $44 \pm 4$ & $0.6 \pm 0.04$ & $0 \pm 0$ \\
\hline P15924 & DSP & $91.83 \pm 7.67$ & $41 \pm 0.57$ & $59 \pm 4$ & $0.58 \pm 0.06$ & $0 \pm 0$ \\
\hline P23246 & SFPQ & $14.35 \pm 0.45$ & $29.35 \pm 2.76$ & $12 \pm 2$ & $0.51 \pm 0.16$ & $0.02 \pm 0.01$ \\
\hline P23381 & WARS & $31.38 \pm 0.58$ & $60.2 \pm 6.79$ & $28 \pm 1$ & $0.45 \pm 0.08$ & $0 \pm 0$ \\
\hline P23396 & RPS3 & $31.17 \pm 2.14$ & $71.85 \pm 3.18$ & $28 \pm 4$ & $0.43 \pm 0.05$ & $0 \pm 0$ \\
\hline P26196 & DDX6 & $27.78 \pm 1.64$ & $45.55 \pm 3.18$ & $24 \pm 2$ & $0.64 \pm 0.01$ & $0.02 \pm 0.01$ \\
\hline P28289 & TM0D1 & $7.16 \pm 1.19$ & $33.85 \pm 1.34$ & $5 \pm 1$ & $0.37 \pm 0.3$ & $0.02 \pm 0.01$ \\
\hline P49411 & TUFM & $40.15 \pm 0.54$ & $61.3 \pm 1.56$ & $39 \pm 6$ & $0.55 \pm 0.05$ & $0.03 \pm 0$ \\
\hline P49588 & AARS & $71.83 \pm 6.77$ & $58.55 \pm 1.77$ & $63 \pm 5$ & $0.43 \pm 0.09$ & $0.01 \pm 0.01$ \\
\hline P49792 & RANBP2 & $59.85 \pm 10.34$ & $29.25 \pm 0.35$ & $38 \pm 5$ & $0.6 \pm 0.05$ & $0.01 \pm 0.01$ \\
\hline P50454 & SERPINH1 & $40.25 \pm 1.66$ & $60.05 \pm 1.06$ & $38 \pm 6$ & $0.55 \pm 0.04$ & $0.01 \pm 0.01$ \\
\hline P52948-6 & NUP98 & $30.96 \pm 2.03$ & $20.45 \pm 0.21$ & $21 \pm 1$ & $0.57 \pm 0.05$ & $0 \pm 0$ \\
\hline P54136 & RARS & $53.15 \pm 2.3$ & $57.8 \pm 0.57$ & $40 \pm 1$ & $0.55 \pm 0.1$ & $0.01 \pm 0.01$ \\
\hline P62249 & RPS16 & $13.46 \pm 0.86$ & $59.9 \pm 2.4$ & $8 \pm 0$ & $0.6 \pm 0.1$ & $0.04 \pm 0.01$ \\
\hline P62805 & HIST1H4A & $8.01 \pm 0.01$ & $39.8 \pm 1.41$ & $9 \pm 3$ & $0.27 \pm 0.02$ & $0.02 \pm 0$ \\
\hline P78527 & PRKDC & $128.53 \pm 8.53$ & $33.15 \pm 0.49$ & $103 \pm 6$ & $0.5 \pm 0.04$ & $0 \pm 0$ \\
\hline Q00839 & HNRNPU & $39.34 \pm 3.51$ & $42.75 \pm 5.73$ & $36 \pm 2$ & $0.58 \pm 0.05$ & $0.04 \pm 0.01$ \\
\hline Q08211 & DHX9 & $63.55 \pm 2.37$ & $44.85 \pm 3.32$ & $51 \pm 7$ & $0.52 \pm 0.04$ & $0.01 \pm 0$ \\
\hline Q13085 & ACACA & $73 \pm 2.45$ & $34.8 \pm 1.56$ & $43 \pm 1$ & $0.52 \pm 0.15$ & $0 \pm 0$ \\
\hline Q13283 & G3BP1 & $21.02 \pm 0.37$ & $41.95 \pm 2.33$ & $21 \pm 1$ & $0.58 \pm 0.02$ & $0 \pm 0$ \\
\hline Q14694-2 & USP10 & $19.38 \pm 5.14$ & $24.1 \pm 3.82$ & $12 \pm 2$ & $0.44 \pm 0.01$ & $0.03 \pm 0.01$ \\
\hline Q14764 & MVP & $41.48 \pm 1.61$ & $47.7 \pm 3.68$ & $29 \pm 2$ & $0.49 \pm 0.04$ & $0 \pm 0$ \\
\hline Q14847 & LASP1 & $16.86 \pm 2.24$ & $76.05 \pm 4.03$ & $23 \pm 2$ & $0.4 \pm 0.03$ & $0.02 \pm 0.02$ \\
\hline Q16555 & DPYSL2 & $37.09 \pm 3.37$ & $59.45 \pm 5.44$ & $41 \pm 6$ & $0.22 \pm 0.05$ & $0 \pm 0$ \\
\hline Q16822 & PCK2 & $21.86 \pm 0.25$ & $32.95 \pm 1.77$ & $13 \pm 1$ & $0.44 \pm 0.06$ & $0.01 \pm 0.01$ \\
\hline Q8IVF2-3 & AHNAK2 & $135.87 \pm 9.67$ & $47.45 \pm 0.64$ & $97 \pm 3$ & $0.33 \pm 0.09$ & $0 \pm 0$ \\
\hline Q92616 & GCN1L1 & $86.69 \pm 1.72$ & $33.9 \pm 1.7$ & $60 \pm 4$ & $0.29 \pm 0.12$ & $0 \pm 0$ \\
\hline Q92945 & KHSRP & $26.77 \pm 3.58$ & $45.9 \pm 2.26$ & $22 \pm 2$ & $0.38 \pm 0.05$ & $0.02 \pm 0.01$ \\
\hline Q96T88 & UHRF1 & $16.55 \pm 3.56$ & $26.65 \pm 4.45$ & $11 \pm 1$ & $0.5 \pm 0.04$ & $0.02 \pm 0$ \\
\hline Q9BUQ8 & DDX23 & $26.14 \pm 0.06$ & $31 \pm 1.98$ & $14 \pm 1$ & $0.56 \pm 0.04$ & $0.02 \pm 0.01$ \\
\hline Q9H0A0 & NAT10 & $25.41 \pm 1.66$ & $31.8 \pm 0.28$ & $17 \pm 1$ & $0.53 \pm 0.01$ & $0.01 \pm 0.01$ \\
\hline Q9Y2T3-3 & GDA & $37.68 \pm 1.19$ & $66.45 \pm 3.32$ & $28 \pm 1$ & $0.33 \pm 0.02$ & $0.04 \pm 0.01$ \\
\hline Q9Y617 & PSAT1 & $32.7 \pm 0.34$ & $70.55 \pm 0.35$ & $30 \pm 5$ & $0.46 \pm 0.07$ & $0 \pm 0$ \\
\hline C9J8U1 & SPECC1L & $13.39 \pm 0.28$ & $33.75 \pm 0.92$ & $9 \pm 1$ & $0.51 \pm 0.04$ & $0.03 \pm 0.01$ \\
\hline F5GWX5 & CHD4 & $44.48 \pm 0.42$ & $28.3 \pm 0.85$ & $29 \pm 2$ & $0.36 \pm 0.03$ & $0 \pm 0$ \\
\hline F5H0L8 & SEC23IP & $23.75 \pm 1.86$ & $38.9 \pm 1.84$ & $14 \pm 2$ & $0.26 \pm 0.04$ & $0.03 \pm 0$ \\
\hline F5H2U2 & PRPF4B & $12.99 \pm 0.78$ & $22.15 \pm 6.58$ & $7 \pm 1$ & $0.41 \pm 0.03$ & $0.01 \pm 0$ \\
\hline I3L4C2 & BAIAP2 & $29.04 \pm 0.88$ & $48.3 \pm 2.26$ & $19 \pm 1$ & $0.45 \pm 0.29$ & $0 \pm 0.01$ \\
\hline J3KTA4 & DDX5 & $38.65 \pm 0.95$ & $50.95 \pm 8.27$ & $32 \pm 0$ & $0.55 \pm 0.05$ & $0.03 \pm 0.01$ \\
\hline J9IID7 & LMNB2 & $14.78 \pm 1.04$ & $33.25 \pm 2.47$ & $9 \pm 1$ & $0.53 \pm 0.16$ & $0 \pm 0$ \\
\hline
\end{tabular}




\begin{tabular}{|c|c|c|c|c|c|c|c|c|c|}
\hline \multirow{6}{*}{ (A) } & & miR-CTL & $\operatorname{miR}-193 \mathrm{a}-3 \mathrm{~F}$ & & \multirow[b]{2}{*}{ (B) } & & \multirow{2}{*}{\multicolumn{2}{|c|}{ miR-CTL miR-193a-3p }} & \multirow[b]{3}{*}{$52 \mathrm{KDa}$} \\
\hline & HNRNPU & - & 5 & $85 \mathrm{KDa}$ & & \multirow{4}{*}{$\begin{array}{c}\text { PPP2R2A } \\
\text { GSN } \\
\beta \text {-actin }\end{array}$} & & & \\
\hline & G3BP1 & & & $52 \mathrm{KDa}$ & & & $=$ & $=$ & \\
\hline & UHRF1 & $=$ & $=$ & $91 \mathrm{KDa}$ & & & $-\cdots$ & - & $83 \mathrm{KDa}$ \\
\hline & LMNB2 & & - & $67 \mathrm{KDa}$ & & & 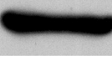 & - & $42 \mathrm{KDa}$ \\
\hline & $\beta$-actin & $=$ & 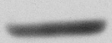 & $42 \mathrm{KDa}$ & & & & & \\
\hline
\end{tabular}

Fig. 3. The expression levels of the dysregulated proteins were verified using WB analysis. (A) Representative blots show the protein expression level in the miR-193a-3p-overexpressing SPC-A-1sci cells. HNRNPU, G3BP1, UHRF1, LMNB2 were down-regulated proteins, and PPP2R2A and GSN were up-regulated proteins (B). $\beta$-actin was used as the loading control.

Fig. 4. The 112 dysregulated proteins were analyzed according to GO terms. (A) All of the dysregulated proteins were analyzed by searching the DAVID database, $\mathrm{P}<0.05$. The histograms demonstrate the top 10 counts for a biological process, a cellular component (B) and a molecular function (C).

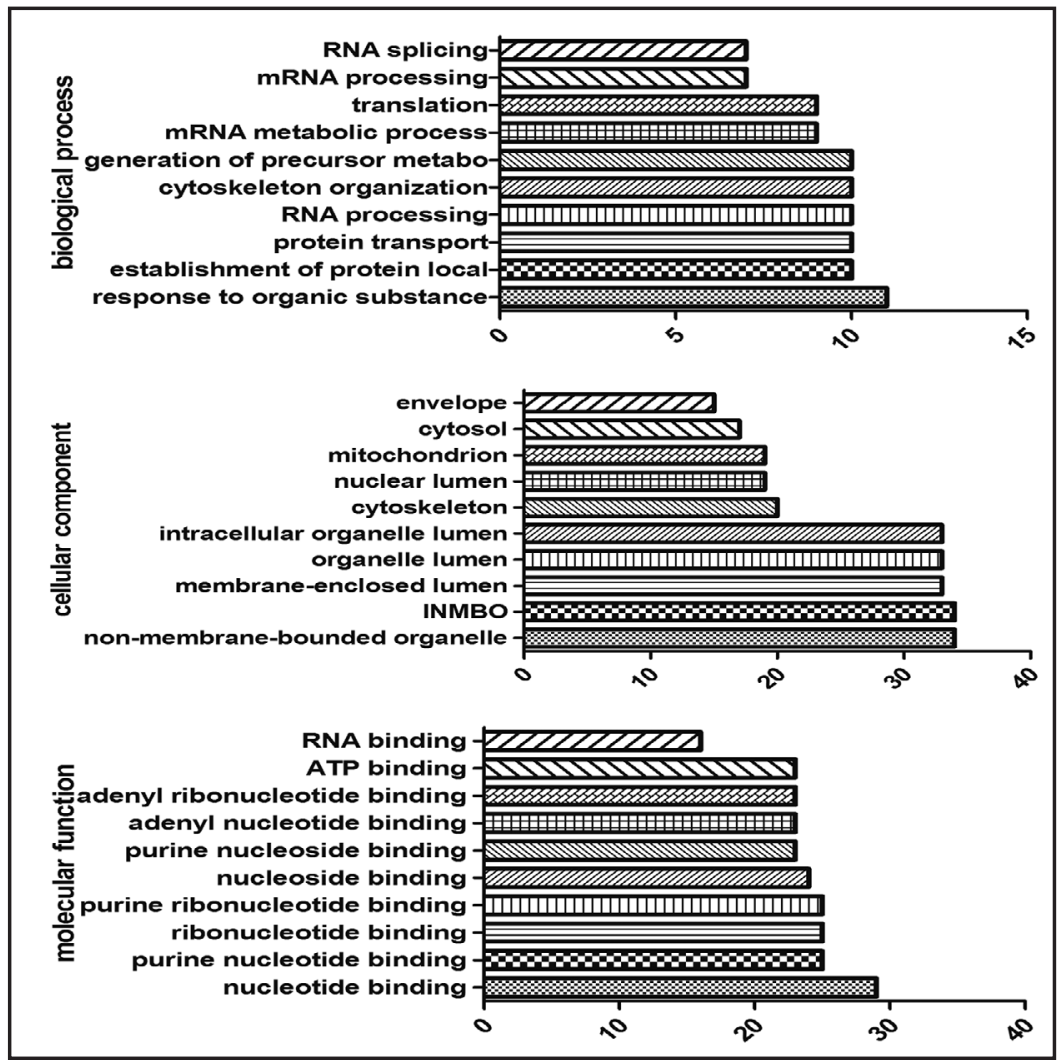

myelogenous leukemia and prostate adenocarcinoma, as well as in lung cancer [26-28].

The proteins with down-regulated expression mediated by the overexpression of miR193a-3p are UHRF1, G3BP1, LMNB2 and HNRNPU.

UHRF1 belongs to the UHRF family and is a nucleoprotein involved in cell growth and proliferation [29]. It was found that UHRF1 could promote the G1/S transition as a direct target of E2F1, which is a transcriptional factor essential for S-phase entry [30]. In examining many human cancers, including lung cancer, breast cancer and colorectal cancer [31-33], we have found that UHRF1 could promote their proliferation and metastasis.

G3BP1 is one member of the G3BP family that participates in several signaling pathways involved in carcinogenesis. G3BP1, which was reported to be highly overexpressed in various cancers, including breast cancer, esophageal squamous carcinoma and lung cancer [34-36], was found to be closely associated with the invasion and metastasis of tumor cells.

LMNB2 is a component of the nuclear lamina, which is the intermediate-filament 


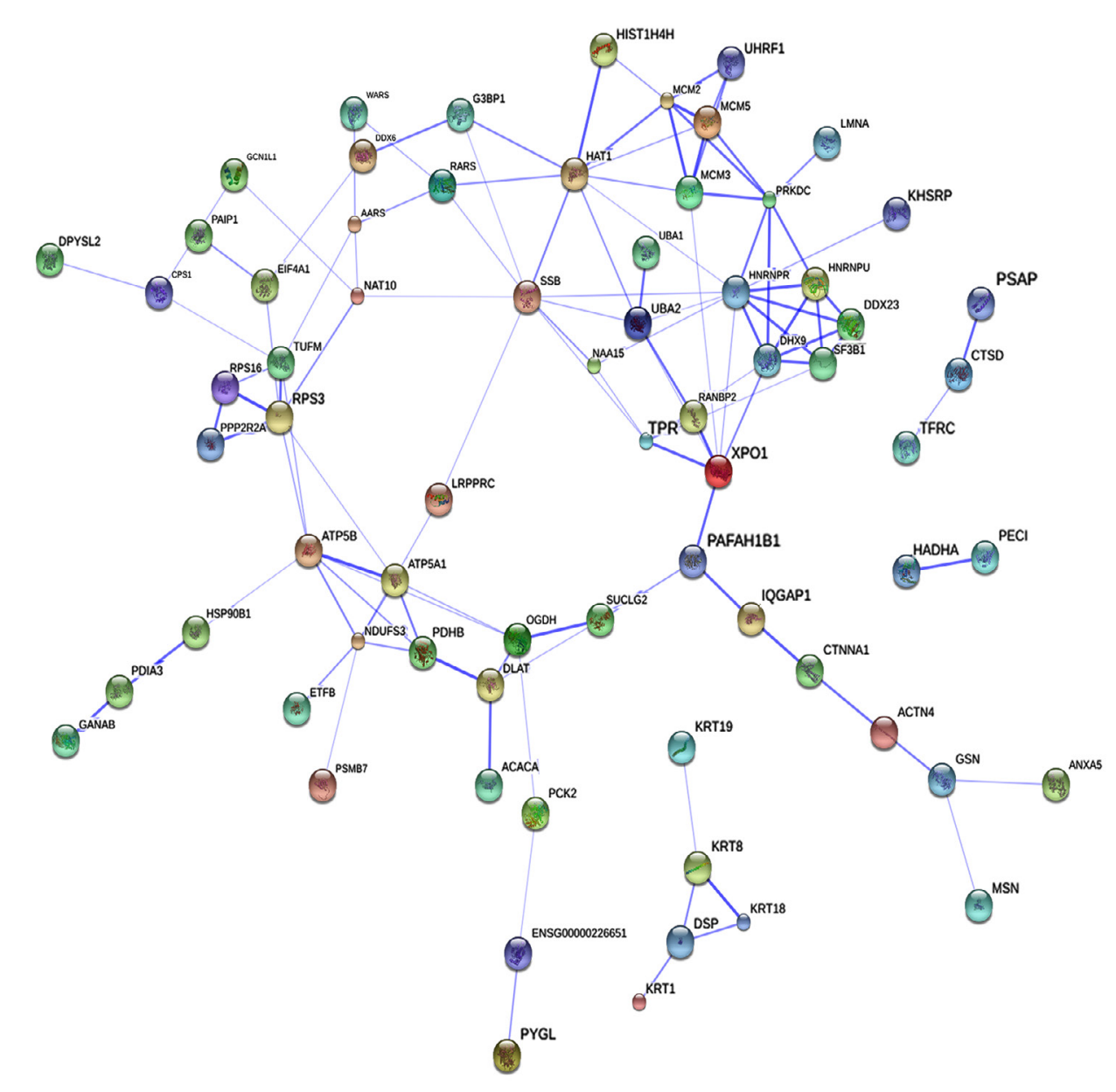

Fig. 5. The differentially expressed proteins were used to search the STRING database to predict their protein-protein interactions in the miR-193a-3p overexpressing SPC-A-1sci cells. In the network shown, the nodes are the proteins and the lines represent the predicted functional associations. The number of lines indicates the strength of the predicted functional interactions of the proteins.

meshwork lying beneath the inner nuclear membrane [37]. HNRNPU, which plays an important role in the processing and transport of mRNA, is the largest member of the hnRNP protein family [38]. LMNB2 and HNRNPU had not been reported in lung cancer progression. They might be new targets of miR-193a-3p-mediated inhibition of SPC-A-1sci migration.

In summary, we used the iTRAQ quantitative approach combined with nano liquid chromatography-tandem mass spectrometry analysis to identify the proteins species with altered levels of expression in SPC-A-1sci cells overexpressing miR-193a-3p. Our findings may improve the understanding of the molecular mechanisms underlying the metastasisinhibitory effects of miR-193a-3p on lung cancer cells.

\section{Acknowledgement}

This work was supported by Shanghai Science and Technology Developing Program (13140900502), and State Key Laboratory of Oncogenes and Related Genes Research Fund (91-14-15). 


\section{Cellular Physiology Cell Physiol Biochem 2015;35:1677-1688

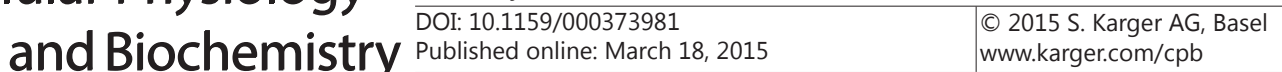

\section{References}

1 Siegel R, Naishadham D, Jemal A: Cancer statistics for Hispanics/Latinos, 2012. CA Cancer J Clin 2012;5:283-298.

2 Crino L, Weder W, van Meerbeeck J, Felip E, Group EGW: Early stage and locally advanced (non-metastatic) non-small-cell lung cancer: ESMO Clinical Practice Guidelines for diagnosis, treatment and follow-up. Ann Oncol 2010;21:v103-115.

3 Hanahan D, Weinberg RA: The hallmarks of cancer. Cell 2000;1:57-70.

4 Gdowicz-Klosok A, Giglok M, Drosik A, Suwinski R, Butkiewicz D: The SIPA1 -313A>G polymorphism is associated with prognosis in inoperable non-small cell lung cancer. Tumor Biol DOI 10.1007/s13277-0142753-8.

-5 Jia D, Yan M, Wang X, Hao X, Liang L, Liu L, Kong H, He X, Li J, Yao M: Development of a highly metastatic model that reveals a crucial role of fibronectin in lung cancer cell migration and invasion. BMC Cancer 2010;10:364.

6 Liang S, Xu Z, Xu X, Zhao X, Huang C, Wei Y: Quantitative proteomics for cancer biomarker discovery. Comb Chem High T Scr 2012;3:221-231.

7 Nikolov M, Schmidt C, Urlaub H: Quantitative mass spectrometry-based proteomics: an overview. Methods Mol Biol 2012;893:85-100.

8 Masui O, White NM, DeSouza LV, Krakovska O, Matta A, Metias S, Khalil B, Romaschin AD, Honey RJ, Stewart R, Pace K, Bjarnason GA, Siu KW, Yousef GM: Quantitative proteomic analysis in metastatic renal cell carcinoma reveals a unique set of proteins with potential prognostic significance. Mol Cell Protemics 2013;1:132-144.

-9 Jeppesen DK, Nawrocki A, Jensen SG, Thorsen K, Whitehead B, Howard KA, Dyrskjot L, Orntoft TF, Larsen MR, Ostenfeld MS: Quantitative proteomics of fractionated membrane and lumen exosome proteins from isogenic metastatic and nonmetastatic bladder cancer cells reveal differential expression of EMT factors. Proteomics 2014;6:699-712.

10 Bartel DP: MicroRNAs: target recognition and regulatory functions. Cell 2009;2:215-233.

11 Selbach M, Schwanhausser B, Thierfelder N, Fang Z, Khanin R, Rajewsky N: Widespread changes in protein synthesis induced by microRNAs. Nature 2008;7209:58-63.

12 Calin GA, Croce CM: MicroRNA-cancer connection: the beginning of a new tale. Cancer Res 2006;15:73907394.

13 Ambros V: The functions of animal microRNAs. Nature 2004;7006:350-355.

14 Shkumatava A, Stark A, Sive H, Bartel DP: Coherent but overlapping expression of microRNAs and their targets during vertebrate development. Gene Dev 2009;4:466-481.

-15 Grimson A, Farh KK, Johnston WK, Garrett-Engele P, Lim LP, Bartel DP: MicroRNA targeting specificity in mammals: determinants beyond seed pairing. Mol Cell 2007;1:91-105.

-16 Chi SW, Zang JB, Mele A, Darnell RB: Argonaute HITS-CLIP decodes microRNA-mRNA interaction maps. Nature 2009;7254:479-486.

17 Ritchie W, Flamant S, Rasko JE: Predicting microRNA targets and functions: traps for the unwary. Nat Methods 2009;6:397-398.

18 Thomson DW, Bracken CP, Goodall GJ: Experimental strategies for microRNA target identification. Nucleic Acids Res 2011;16:6845-6853.

-19 Yu T, Li J, Yan M, Liu L, Lin H, Zhao F, Sun L, Zhang Y, Cui Y, Zhang F, Li J, He X, Yao M: MicroRNA-193a-3p and $-5 p$ suppress the metastasis of human non-small-cell lung cancer by downregulating the ERBB4/ PIK3R3/mTOR/S6K2 signaling pathway. Oncogene DOI: 10.1038/onc.2013.574.

20 Baig RM, Mahjabeen I, Sabir M, Masood N, Ali K, Malik FA, Kayani MA: Mutational spectrum of Gelsolin and its down regulation is associated with breast cancer. Dis Markers 2013;2:71-80.

21 Shieh DB, Chen IW, Wei TY, Shao CY, Chang HJ, Chung CH, Wong TY, Jin YT: Tissue expression of gelsolin in oral carcinogenesis progression and its clinicopathological implications. Oral Oncol 2006;6:599-606.

22 Noske A, Denkert C, Schober H, Sers C, Zhumabayeva B, Weichert W, Dietel M, Wiechen K: Loss of Gelsolin expression in human ovarian carcinomas. Eur J Cancer 2005;3:461-469.

23 Kim JH, Choi YK, Kwon HJ, Yang HK, Choi JH, Kim DY: Downregulation of gelsolin and retinoic acid receptor beta expression in gastric cancer tissues through histone deacetylase 1. J Gastroen Hepatol 2004;2:218224. 


\section{Cellular Physiology Cell Physiol Biochem 2015;35:1677-1688

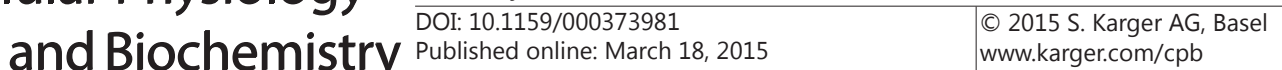

24 Gay F, Estornes Y, Saurin JC, Joly-Pharaboz MO, Friederich E, Scoazec JY, Abello J: In colon carcinogenesis, the cytoskeletal protein gelsolin is down-regulated during the transition from adenoma to carcinoma. Hum Pathol 2008;10:1420-1430.

25 Cheng Y, Liu W, Kim ST, Sun J, Lu L, Sun J, Zheng SL, Isaacs WB, Xu J: Evaluation of PPP2R2A as a prostate cancer susceptibility gene: a comprehensive germline and somatic study. Cancer Genet-NY 2011;7:375381.

-26 Ruvolo PP, Qui YH, Coombes KR, Zhang N, Ruvolo VR, Borthakur G, Konopleva M, Andreeff M, Kornblau SM: Low expression of PP2A regulatory subunit B55alpha is associated with T308 phosphorylation of AKT and shorter complete remission duration in acute myeloid leukemia patients. Leukemia 2011;11:1711-1717.

27 Liu W, Xie CC, Zhu Y, Li T, Sun J, Cheng Y, Ewing CM, Dalrymple S, Turner AR, Sun J, Isaacs JT, Chang BL, Zheng SL, Isaacs WB, Xu J: Homozygous deletions and recurrent amplifications implicate new genes involved in prostate cancer. Neoplasia 2008;8:897-907.

28 Shen S, Yue H, Li Y, Qin J, Li K, Liu Y, Wang J: Upregulation of miR-136 in human non-small cell lung cancer cells promotes Erk1/2 activation by targeting PPP2R2A. Tumor Biol 2014;1:631-640.

29 Jenkins Y, Markovtsov V, Lang W, Sharma P, Pearsall D, Warner J, Franci C, Huang B, Huang J, Yam GC, Vistan JP, Pali E, Vialard J, Janicot M, Lorens JB, Payan DG, Hitoshi Y: Critical role of the ubiquitin ligase activity of UHRF1, a nuclear RING finger protein, in tumor cell growth. Mol Biol Cell 2005;12:5621-5629.

30 Unoki M, Nishidate T, Nakamura Y: ICBP90, an E2F-1 target, recruits HDAC1 and binds to methyl-CpG through its SRA domain. Oncogene 2004;46:7601-7610.

-31 Daskalos A, Oleksiewicz U, Filia A, Nikolaidis G, Xinarianos G, Gosney JR, Malliri A, Field JK, Liloglou T: UHRF1-mediated tumor suppressor gene inactivation in nonsmall cell lung cancer. Cancer 2011;5:10271037.

-32 Li X-L: Exogenous expression of UHRF1 promotes proliferation and metastasis of breast cancer cells. Oncol Rep 2012;28:375-83.

33 Wang F, Yang YZ, Shi CZ, Zhang P, Moyer MP, Zhang HZ, Zou Y, Qin HL: UHRF1 promotes cell growth and metastasis through repression of p16(ink(4)a) in colorectal cancer. Ann Surg Oncol 2012;8:2753-2762.

-34 French J, Stirling R, Walsh M, Kennedy HD: The expression of Ras-GTPase activating protein SH3 domainbinding proteins, G3BPs, in human breast cancers. Histochemical J 2002;5:223-231.

-35 Zhang HZ, Liu JG, Wei YP, Wu C, Cao YK, Wang M: Expression of G3BP and RhoC in esophageal squamous carcinoma and their effect on prognosis. World J Gastroentero 2007;30:4126-4130.

- 36 Zhang H, Zhang SH, He HW, Zhang CX, Yu DK, Shao RG: Downregulation of G3BPs inhibits the growth, migration and invasion of human lung carcinoma H1299 cells by suppressing the Src/FAK-associated signaling pathway. Cancer Gene Ther 2013;11:622-629.

- 37 Yang SH, Chang SY, Yin L, Tu Y, Hu Y, Yoshinaga Y, de Jong PJ, Fong LG, Young SG: An absence of both lamin B1 and lamin B2 in keratinocytes has no effect on cell proliferation or the development of skin and hair. Hum Mol Genet 2011;18:3537-3544.

-38 Spraggon L, Dudnakova T, Slight J, Lustig-Yariv O, Cotterell J, Hastie N, Miles C: hnRNP-U directly interacts with WT1 and modulates WT1 transcriptional activation. Oncogene 2007;10:1484-1491. 